\title{
Simulación de los subprocesos de llenado, tapado y pasteurizado para la automatización de una planta cervecera
}

\author{
Simulation of the subprocesses of filling, capping and pasteurized for the automation of a \\ brewery
}

\section{Simulação dos processos de enchimento, tapado e pasteurizado para a automatização de uma cervejaria}

\author{
Tatiana Ortegón-Sarmiento ${ }^{1}$, Carol Serrano-Acuña ${ }^{2}$
}

Forma de citar: T. Ortegón-Sarmiento, C. Serrano-Acuña, "Simulación de los subprocesos de llenado, tapado y pasteurizado para la automatización de una planta cervecera", Respuestas, vol. 21, no. 2, pp. 57-70, 2016.

Recibido:

Diciembre 17 de 2015

Aceptado:

Mayo 31 de 2016
${ }^{1}$ Ingeniero en Mecatrónica taxipiorsa@hotmail.com Orcid: 0000-0002-7208-4595

Universidad Militar Nueva Granada

Bogotá D.C.- Colombia

${ }^{2}$ Ingeniero en Mecatrónica u1801965@unimilitar.edu.co Orcid: 0000-0002-8004-1464

Universidad Militar Nueva Granada

Bogotá D.C.- Colombia

\section{Resumen}

Antecedentes: La simulación de procesos es una herramienta útil en el diseño, análisis y evaluación de estos, facilitando así su optimización y monitoreo, y permitiendo la predicción de su comportamiento ante ciertas situaciones. Objetivo: Por lo anterior, en este artículo se presenta la simulación de un proceso cervecero, haciendo especial énfasis en los subprocesos de llenado, tapado y pasteurizado. Método: Se identificaron cada uno de los pasos de un proceso cervecero, y se caracterizaron los subprocesos de interés, definiendo cada una de las variables de entrada y salida. A continuación, se simularon los subprocesos haciendo uso del controlador lógico programable PLC S7-1500, y se supervisó el comportamiento de cada uno de estos mediante SIMATIC WinCC, de manera local en una pantalla HMI (Interfaz Hombre Máquina), y de manera remota en un ordenador. La dinámica del proceso se presenta en el software Automation Studio 6, el cual se conecta al PLC mediante el servidor OPC de IBH. Para observar el comportamiento del sistema ante ciertas situaciones de emergencia se implementaron alarmas. Resultados: La simulación realizada permite seguir paso a paso los subprocesos de interés, tanto de manera local como remota, permitiendo observar el comportamiento del sistema en condiciones normales o ante situaciones de emergencia a las cuales el simulador responde de manera inmediata. Conclusiones: Con base en los resultados obtenidos, se concluye que el simulador es una alternativa adecuada y de bajo costo que permite comprender, supervisar y predecir el comportamiento que tendría la planta real, en cuanto a los subprocesos de interés.

Palabras Clave: Automatización, Control Automático, Controlador Lógico Programable, Diseño de Sistemas, Programación, Simulación de Procesos.

\begin{abstract}
Background: Process simulation is an useful tool in the design, analysis and evaluation of processes, facilitating its optimization and monitoring, and allowing the prediction of its behavior in certain situations. Objective: Therefore, this paper shows a simulation of a brewing process with special emphasis on the subprocesses of filling, capping and pasteurized. Method: Each of the steps of a brewing process was identified, and the subprocesses of interest were characterized, defining each of the input and output variables.
\end{abstract}


No. 2

Jul - Dic 2016

ISSN 0122-820X

E-ISSN 2422-5053

PP: $57-70$
For the simulation of the subprocesses, a programmable logic controller PLC S7-1500 was used, controlling and monitoring the behavior of each of these by SIMATIC WinCC, locally on an HMI (Human Machine Interface) screen and remotely on a computer. The dynamics of the process are displayed in the Automation Studio 6 software, which is connected to the PLC via the IBH OPC server. To observe the behavior of the system to certain emergency situations alarms were implemented. Results: The performed simulation, allowed to follow the subprocesses of interest step by step, both locally and remotely, allowing to observe the system behavior under normal conditions or emergency situations, when the simulator responds immediately. Conclusions: Based on the results obtained, it is concluded that the simulator is an adequate and inexpensive alternative for understanding, monitoring and predicting the behavior that would have the real plant in terms of the subprocesses of interest.

Keywords: Automatic Control, Automation, Process Simulation, Programmable Logic Controller, Programming, Systems Design.

\section{Resumo}

Antecedentes: A simulação de processos é uma ferramenta útil na concepção, análise a avaliação destes, facilitando assim sua optimização e monitoramento, permitindo a predição do seu comportamento em determinadas situações. Objetivo: Portanto, neste artigo se apresenta a simulação de um processo cervejeiro, fazendo especial ênfase nos processos de enchimento, tapado e pasteurizado. Metodologia: Identificaram-se cada um dos passos de um processo cervejeiro, e caracterizaram-se os processos de interesse, definindo cada uma das variáveis de entrada e saída. A continuação, se simularam os processos fazendo uso do controlador lógico programável PLC S7-1500, e se supervisou o comportamento de cada um destes através de SIMATIC WinCC, localmente numa tela HMI (Interface Homem-Máquina), e remotamente em um computador. A dinâmica do processo se apresenta no software Automation Studio 6, que está ligado ao PLC através do servidor OPC de IBH. Para observar o comportamento do sistema em certas situações de emergência, foram implementados alarmes. Resultados: A simulação realizada permitiu acompanhar passo a passo os processos de interesse, tanto local como remotamente, permitindo observar o comportamento do sistema em condições normais ou situações de emergência nas quais o simulador responde imediatamente. Conclusões: Com base nos resultados obtidos, conclui-se que o simulador é uma alternativa adequada e de baixo custo que permite compreender, acompanhar e prever o comportamento que poderia ter a planta real no que respeita aos processos de interesse.

Palavras-chave: Automatização, Controle Automático, Controlador Lógico Programável, Design de Sistemas, Programação, Simulação de Processos.

\section{Introducción}

La automatización es un área de la ingeniería que consiste en el control computarizado y electromecánico de procesos industriales o maquinarias [1], la cual surge de la necesidad de mejorar, aumentar, y optimizar la producción de una fábrica con costos más eficientes [2]. Ésta ha ido ganando gran importancia en la industria a lo largo del tiempo, debido a que permite reducir costos, mejorar la calidad de los productos, maximizar la producción, brindar una mayor seguridad a los trabajadores, y controlar y supervisar todos los sistemas de una planta [3]. 
Para la automatización de procesos se suelen usar distintos elementos, como es el caso de los PLC, los cuales son dispositivos electrónicos que gobiernan procesos lógicos y/o secuenciales [4]. Las ventajas de estos dispositivos radican en que usan poco cableado, emplean un menor espacio físico, son fáciles de programar, son flexibles, y el mantenimiento de estos es fácil. En [5] se describe como se emplea un sistema de control basado en PLC para realizar la automatización de los procesos y plantas de gran escala de una de las mayores compañias cerveceras de Japón.

La simulación de procesos sirve como herramienta de apoyo para la automatización, en cuanto a que permite reproducir de manera virtual los procesos, facilitando así la observación y evaluación del comportamiento de estos, de manera que el usuario pueda anticiparse al proceso real y seleccionar la configuración mas adecuada, en cuanto a costos, eficiencia y productividad [6].

En la actualidad los procesos industriales demandan sistemas de automatización altamente confiables para una operación clara, objetiva y de calidad [7], sin ser esto la excepción para las plantas cerveceras, unas de las más importantes en la industria de los alimentos.

El proceso de producción de la cerveceza se divide en cuatro subprocesos: elaboración del mosto, fermentación y maduración, filtración y almacenamiento, y envasado, dichos subprocesos cuentan con una gran cantidad de variables de entrada y salida, las cuales hacen de esta industria un proceso complejo [5]. Debido a esa complejidad, se han ido desarrollando diferentes sistemas de automatización y simulación para el control de este proceso, siendo este el caso de [8], en el cual se diseña e implementa un sistema de control basado en PLC para el proceso de fermentación. Dichos sistemas, con el paso de los años y el desarrollo de nuevas tecnológias, han ido adquiriendo mayor fuerza, haciendo de la industria cervecera una de las más competitivas, especialmente en Colombia [9].

Considerando lo anterior, en este trabajo se optó por simular los subprocesos de llenado, tapado y pasteurizado, pertenecientes a la etapa de envase de un proceso cervecero, presentando el proceso completo en una pantalla HMI para su posterior monitoreo y control, a fin de predecir y evaluar el comportamiento de un futura planta de cerveza.

Para comenzar, en este artículo se realiza una descripción del proceso efectuado, detallando cada uno de los subprocesos de interés en cuanto a las variables que interfieren y las salidas de estas, y el diseño e implementacion de las secuencias para la simulación y visualización de dichas entradas y salidas. De igual forma se describe el sistema implementado para la simulación de emergencias y la detección de riesgos. Se presentan los resultados, y finalmente las conclusiones.

\section{Materiales y métodos}

El sistema completo se divide en tres subprocesos, llenado, tapado y pasteurizado, a los cuales el usuario puede acceder por medio de un menú presentado tanto en una HMI como en el ordenador. Para comenzar el proceso de simulación, se debe activar un botón de inicio en la pantalla de visualización del procedimiento, así como la banda transportadora encargada de llevar las botellas. Dicha banda estará en constante movimiento durante los subprocesos, a menos que se detenga con el interruptor de esta. Una vez que se inició el proceso, el usuario accede a la etapa de llenado, en la cual, se despliega la simulación de ésta en la pantalla HMI. Al terminar esta etapa el 
No. 2

Jul - Dic 2016

ISSN 0122-820X

E-ISSN 2422-5053

PP: $57-70$ usuario puede acceder a la parte del tapado de las botellas, donde, al igual que en la anterior etapa, se muestra la simulación de ésta. A continuación, se presenta la simulación de la etapa de pasteurizado, en la que el usuario puede monitorear la temperatura del agua de pasteurizado. Al mismo tiempo que se ejecutan las simulaciones de cada uno de los subprocesos en la HMI, en el software Automation Studio 6 se ejecuta la dinámica del sistema. En la Figura 1 se presentan los tres subprocesos.

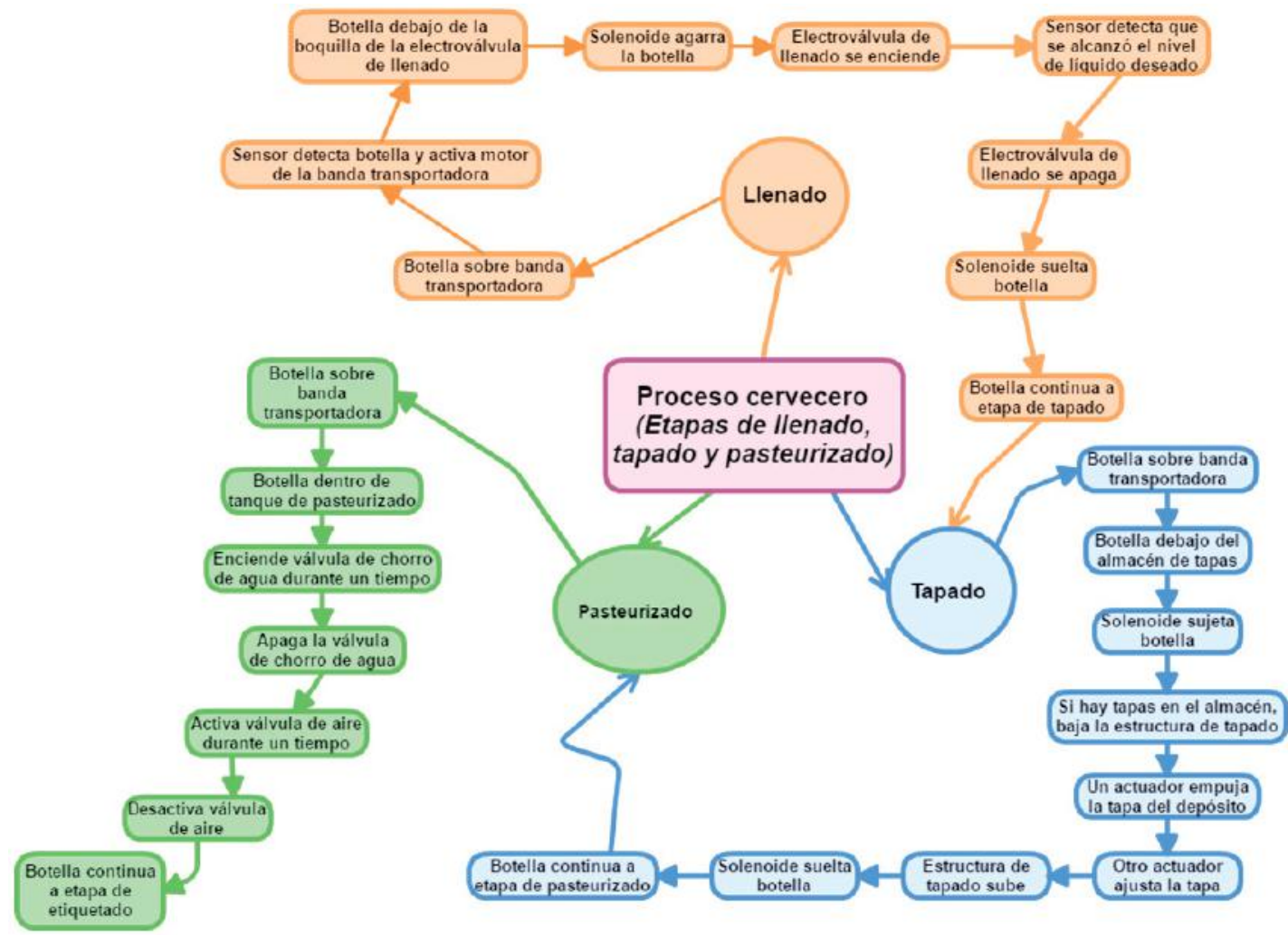

Figura 1. Diagrama de los subprocesos de llenado, tapado y pasteurizado implementado en Cacoo Fuente: Autores

El usuario puede acceder a una etapa de monitoreo, donde se presentan las distintas alarmas que posee el sistema. De igual forma, a fin de observar la respuesta del sistema a ciertos escenarios de emergencia, el usuario puede activar ciertas situaciones que alterarán el proceso, y provocarán que se activen las alarmas.

Para lograr lo anterior, el trabajo contó con un sistema de adquisición de datos para la supervisión y el control de procesos a distancia, es decir, un sistema SCADA. Así mismo, este contó con un sistema de control de velocidad para el caso de la banda transportadora, un sistema de control de temperatura del agua para la etapa de pasteurizado, y un servidor OPC de IBH para el envío de los datos de simulación de las distintas etapas al software Automation Studio. Los sensores distribuidos a lo largo de todo el proceso, se activan y desactivan mediante control on/off.

\subsection{Subproceso de llenado}

En este subproceso se llenan las botellas de cerveza hasta un nivel predefinido. A continuación, en la Tabla I se presentan las variables que interfieren en el subproceso: 
Tabla I. Descripción de las variables que interfieren en el subproceso de llenado

\begin{tabular}{|l|l|}
\hline \multicolumn{1}{|c|}{ ENTRADA } & \multicolumn{1}{|c|}{ SALIDA } \\
\hline 6- Botella en plataforma & $\begin{array}{l}\text { B-Cilindro de la plataforma empuja botella } \\
\text { a la banda transportadora }\end{array}$ \\
\hline 1- Botella en banda & $\begin{array}{l}\text { C-Se abre compuerta que permite a botella } \\
\text { pasar a la siguiente etapa (válvula llenado) }\end{array}$ \\
\hline 2- Botella bajo válvula de llenado & $\begin{array}{l}\text { D-Solenoide agarra botella } \\
\text { TON1- Se activa temporizador } \\
\text { Esta salida se desactiva si el sensor que } \\
\text { detecta el nivel de llenado, está activado }\end{array}$ \\
\hline $\begin{array}{l}\text { 3- Líquido en depósito } \\
\text { TON 1 }\end{array}$ & $\begin{array}{l}\text { E-Activa válvula de llenado. } \\
\text { Esta salida se desactiva si el sensor que } \\
\text { detecta el nivel de llenado, está activado }\end{array}$ \\
\hline
\end{tabular}

Fuente: Autores.

En la Figura 2 se presenta la simulación de esta etapa, con los números y letras correspondientes a cada una de las variables de entrada y salida.

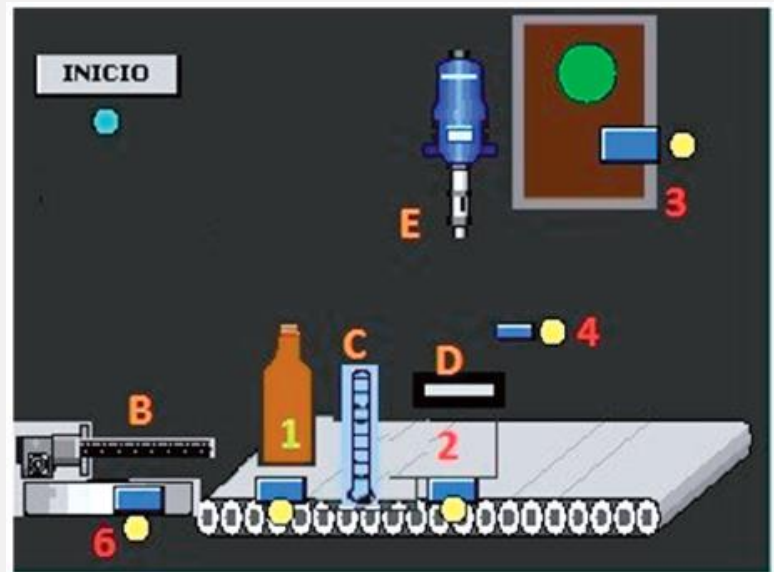

Figura 2. Simulación del subproceso de llenado de una botella

Fuente: Autores

Para el diseño de esta etapa se realizó un grafcet donde se presenta la secuencia a ejecutar. En la Figura 3 (a) se muestra el grafcet simulado en el software FluidSIM, junto con las entradas y salidas de este, presentadas en la figura 3 (b).

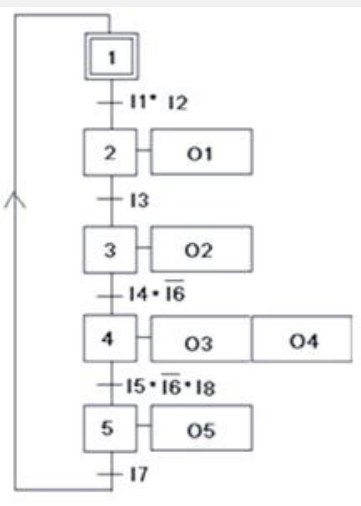

(a)

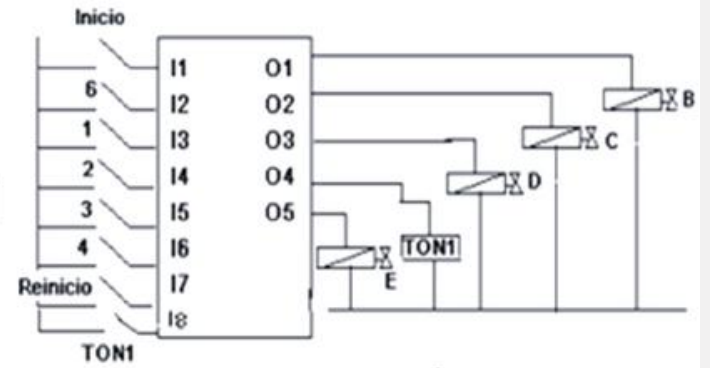

(b)

Figura 3. (a) Grafcet de la secuencia del subproceso de llenado. (b)entradas y salidas del Grafcet Fuente: Autores 
Finalmente, para simular, y ejecutar en el PLC esta etapa, se implementó la secuencia anterior en el programa TIA Portal en lenguaje ladder, parte de la secuencia se presenta en la Figura 4.

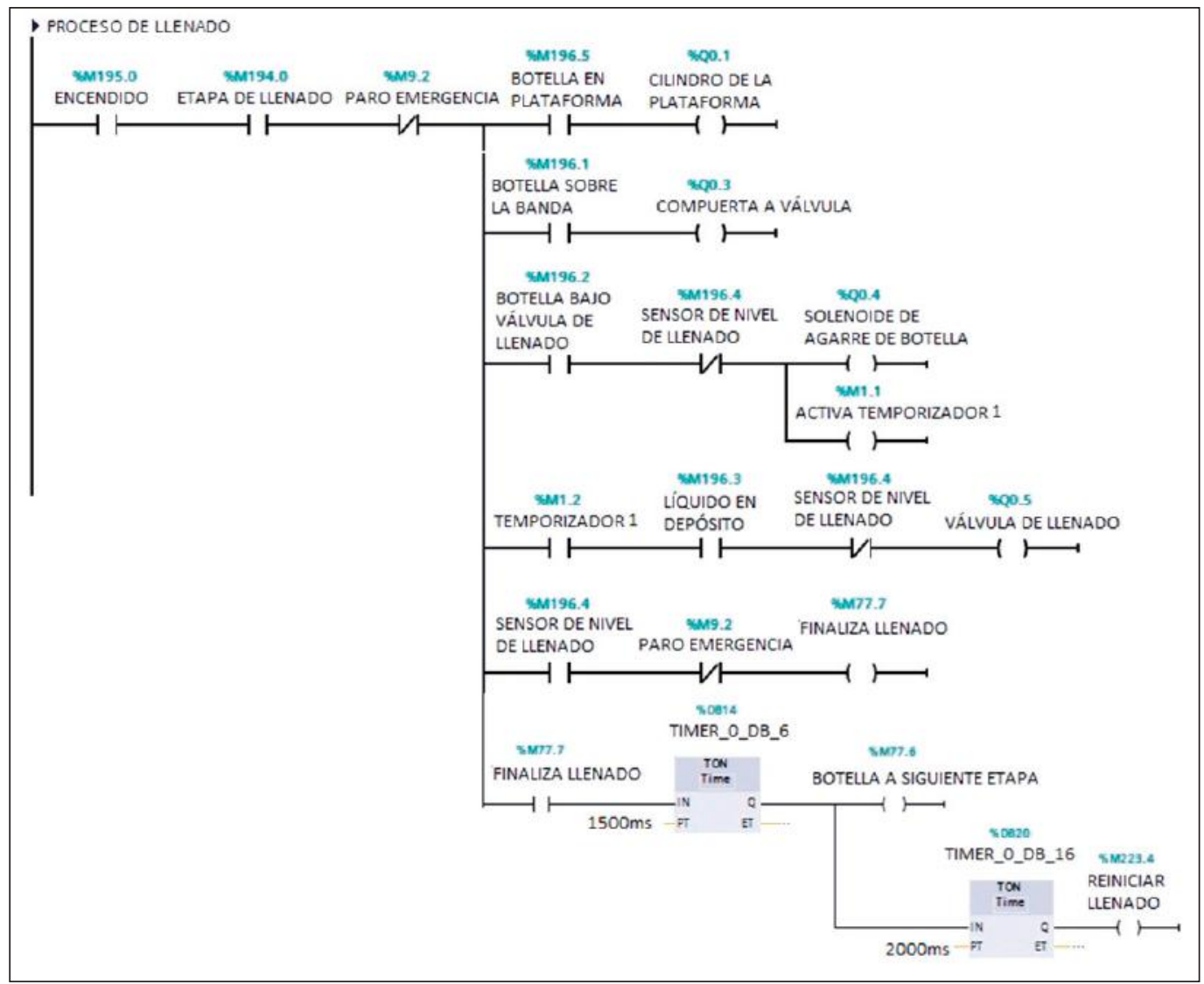

Figura 4. Ladder implementado en el TIA Portal para el subproceso de llenado Fuente: Autores

\subsection{Subproceso de tapado}

En este subproceso se cierran las botellas de cerveza con las tapas que se encuentran en el depósito. A continuación, en la Tabla II se presentan las variables que interfieren en el subproceso:

Tabla II. Descripción de las variables que interfieren en el subproceso de tapado

\begin{tabular}{|l|l|}
\hline \multicolumn{1}{|c|}{ ENTRADA } & \multicolumn{1}{c|}{ SALIDA } \\
\hline 1- Botella en banda & $\begin{array}{l}\text { A-Se abre compuerta que permite a botella } \\
\text { pasar a la siguiente etapa (estructura tapa) }\end{array}$ \\
\hline 2- Botella bajo depósito de tapado & B-Solenoide agarra botella \\
\hline $\begin{array}{l}\text { 3- Tapas depósito } \\
\text { B-Solenoide }\end{array}$ & Cb-Estructura baja \\
\hline 4-Estructura abajo & $\begin{array}{l}\text { D-Cilindro tapas } \\
\text { TON2 -Se activa temporizador }\end{array}$ \\
\hline TON2 & E- Actuador ajusta tapa a botella \\
\hline E-Actuador tapa & TON3 -Se activa temporizador \\
\hline TON3 & 7-Sensor detecta que se colocó la tapa \\
\hline 7- Sensor detecta que se colocó la tapa & TON4 -Se activa temporizador \\
\hline TON4 & Ca-Estructura asciende \\
\hline
\end{tabular}

Fuente: Autores

En la Figura 5 se presenta la simulación de esta etapa, con los números y letras correspondientes a cada una de las variables de entrada y salida. 


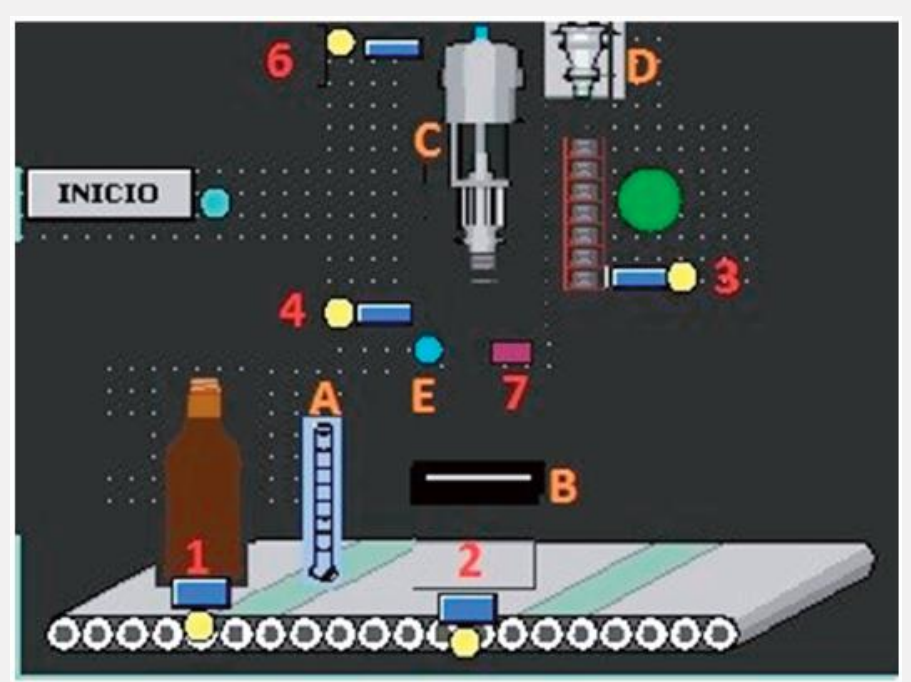

Figura 5. Simulación del subproceso de tapado de una botella Fuente: Autores.

Para el diseño de esta etapa se realizó un grafcet donde se presenta la secuencia a ejecutar. En la Figura 6 (a) se muestra el grafcet simulado en el software FluidSIM, junto con las entradas y salidas de este, presentadas en la Figura 6 (b).

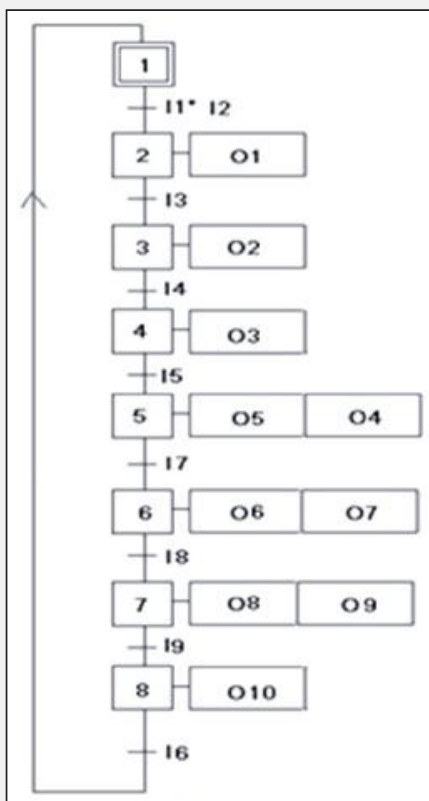

(a)

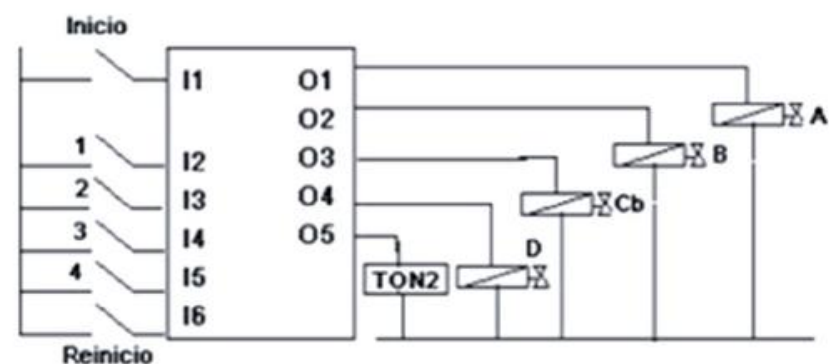

Reinicio

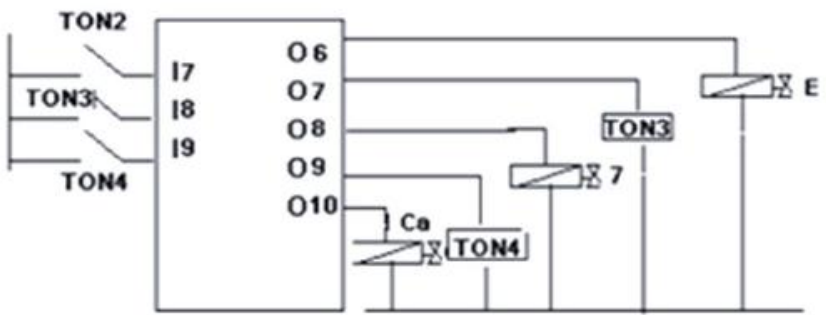

(b)

Figura 6. (a) Grafcet de la secuencia del subproceso de tapado. (b) Entradas y salidas del grafcet Fuente: Autores

Finalmente, para simular, y ejecutar en el PLC esta etapa, se implementó la secuencia anterior en el programa TIA Portal en lenguaje ladder, parte de la secuencia se presenta en la Figura 7. 
Vol. 21

No. 2

Jul - Dic 2016 ISSN 0122-820X E-ISSN 2422-5053 PP: $57-70$

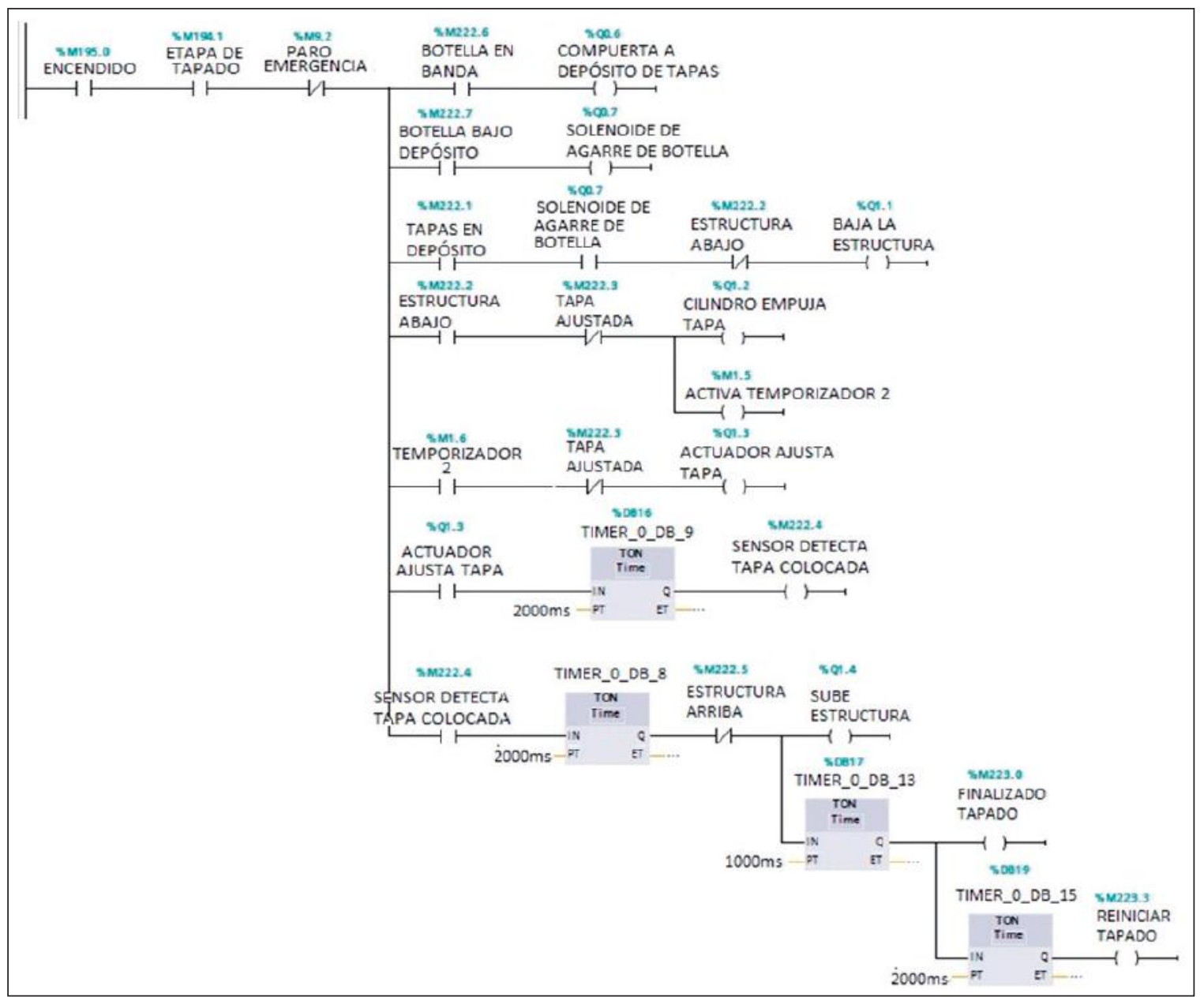

Fuente: Autores

\subsection{Subproceso de pasteurizado}

En este subproceso, se someten las botellas con cerveza a una temperatura de $62^{\circ} \mathrm{C}$ durante unos segundos, seguido de su enfriamiento. Lo anterior se hace con el objetivo de destruir los gérmenes y ampliar el periodo de conservación de la bebida.

A continuación, en la Tabla III se presentan las variables que interfieren en el subproceso:

Tabla III. Descripción de las variables que interfieren en el subproceso de pasteurizado

\begin{tabular}{|l|l|}
\hline \multicolumn{1}{|c|}{ ENTRADA } & \multicolumn{1}{|c|}{ SALIDA } \\
\hline 1- Botella en banda & $\begin{array}{l}\text { A- Se abre compuerta que permite a botella } \\
\text { pasar a la siguiente etapa (pasteurizado) }\end{array}$ \\
\hline 2- Botella en tanque de pasteurizado & $\begin{array}{l}\text { C- Se activa chorro de agua } \\
\text { TON5 -Se activa temporizador } \\
\text { TON6 -Se activa temporizador }\end{array}$ \\
\hline TON5 & Cn-Se desactiva chorro de agua \\
\hline TON6 & $\begin{array}{l}\text { D- Se activa aire para secado } \\
\text { TON7 -Se activa temporizador }\end{array}$ \\
\hline TON7 & $\begin{array}{l}\text { Dn-Se desactiva aire } \\
\text { B- Se abre compuerta que permite a botella } \\
\text { terminar etapa }\end{array}$ \\
\hline
\end{tabular}

Fuente: Autores 
En la Figura 8 se presenta la simulación de esta etapa, con los números y letras correspondientes a cada una de las variables de entrada y salida.

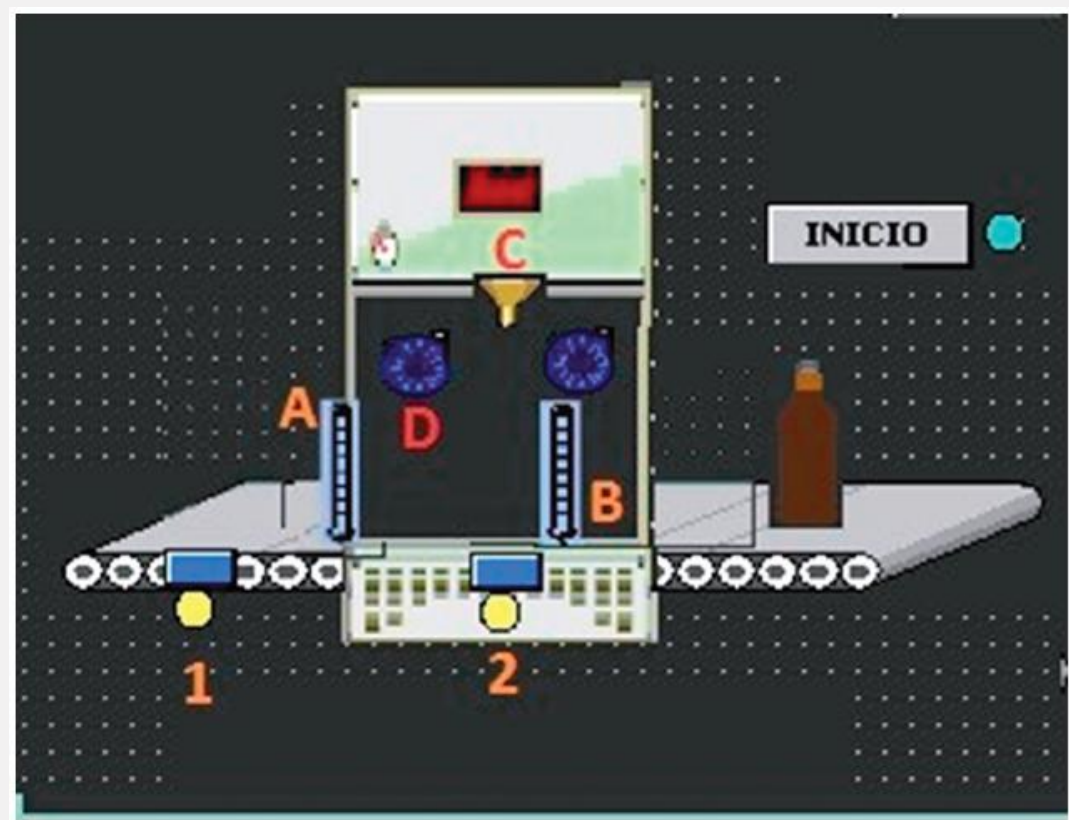

Figura 8. Simulación del subproceso de pasteurizado Fuente: Autores

Para el diseño de esta etapa se realizó un grafcet donde se presenta la secuencia a ejecutar. En la Figura 9 (a) se muestra el grafcet simulado en el software FluidSIM, junto con las entradas y salidas de este, presentadas en la Figura 9 (b).

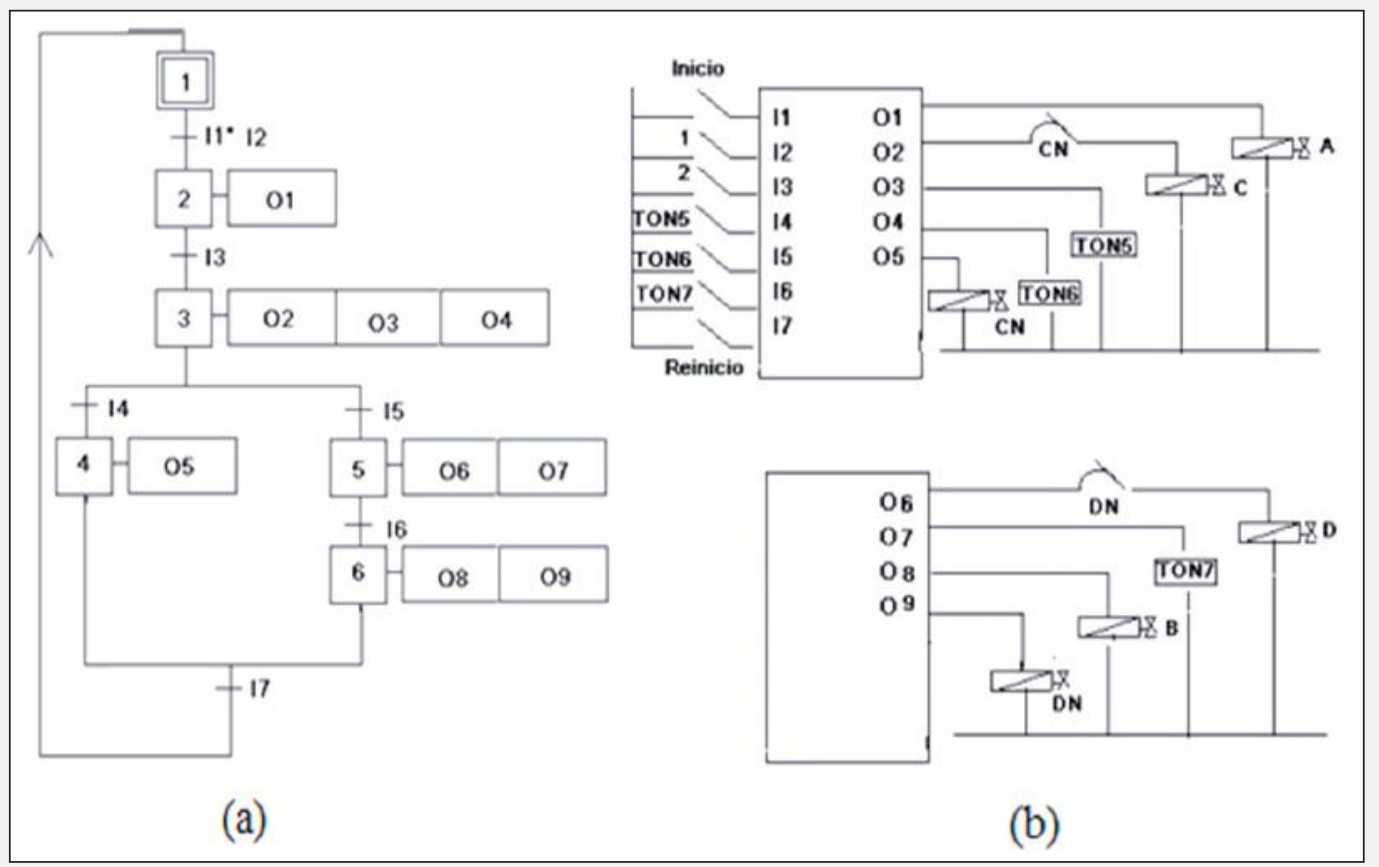

Figura 9. (a) Grafcet de la secuencia del subproceso de pasteurizado. (b) Entradas y salidas del grafcet Fuente: Autores 
Vol. 21

No. 2

Jul - Dic 2016 ISSN 0122-820X E-ISSN 2422-5053 PP: 57-70
Finalmente, para simular, y ejecutar en el PLC esta etapa, se implementó la secuencia anterior en el programa TIA Portal en lenguaje ladder, parte de la secuencia se presenta en la Figura 10.

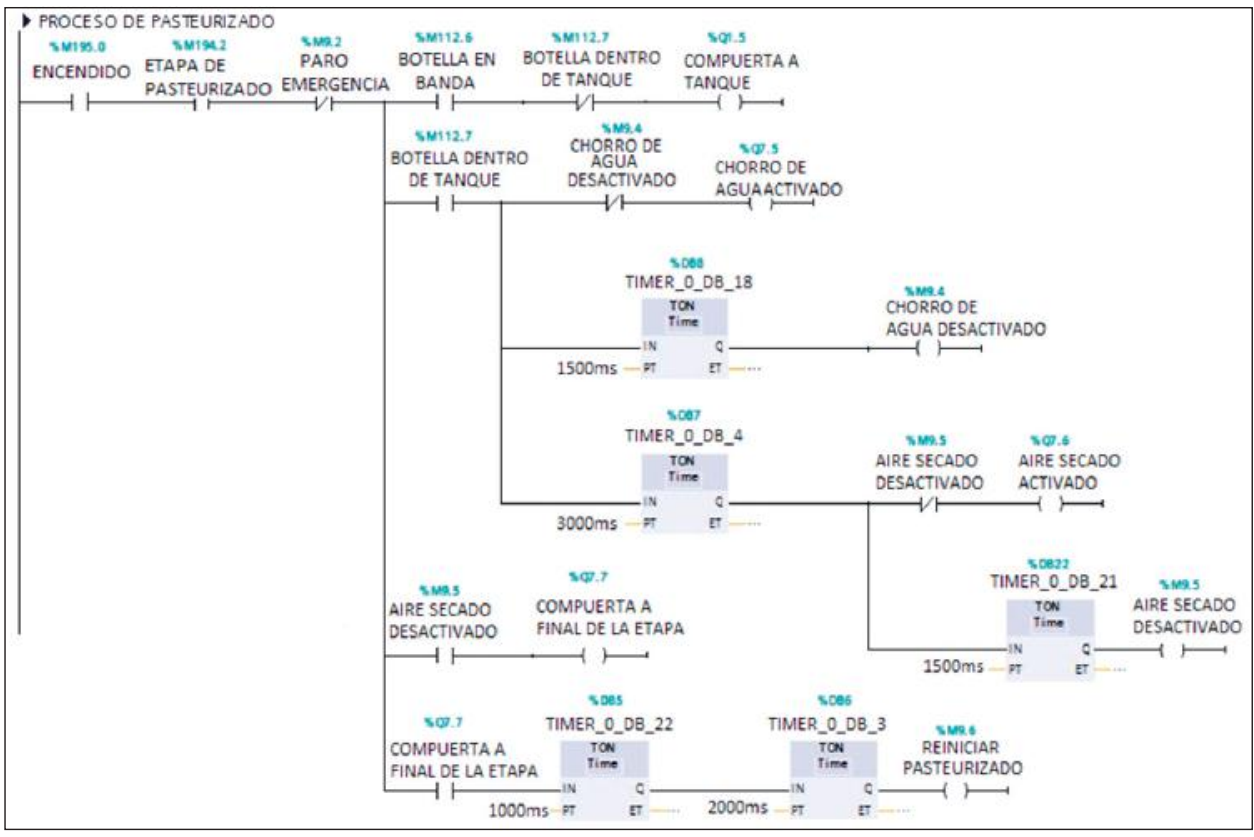

Figura 10. Ladder implementado en el TIA Portal para el subproceso de pasteurizado Fuente: Autores.

\subsection{Alarmas}

\subsubsection{Alarmas digitales}

Se implementaron tres alarmas digitales, mostradas en la Figura 11, las cuales se describen a continuación. En la HMI se pueden visualizar cuales alarmas están activadas, por medio de un bombillo rojo, y cuáles no, por medio de un bombillo verde.

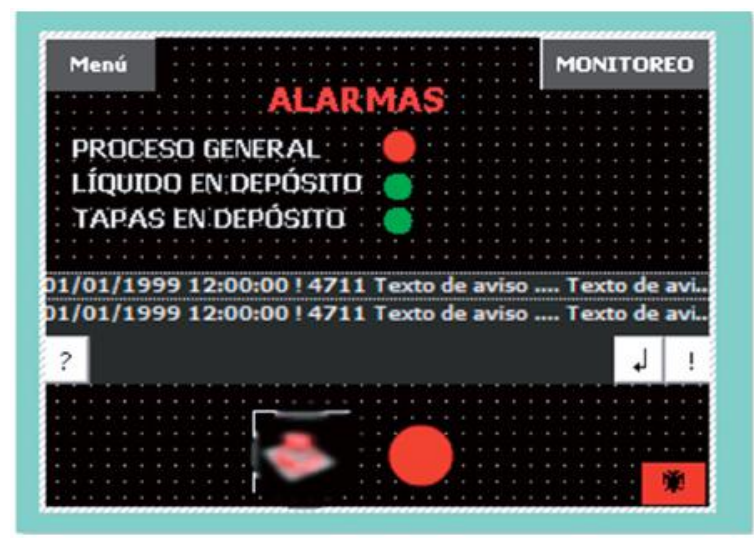

Figura 11. Alarmas digitales del proceso cervecero simulado Fuente: Autores

\section{- Paro de emergencia}

Al activarlo, todo el proceso se detiene, deshabilitando las entradas y salidas. Cuando este se activa se visualiza en la pantalla HMI un botón rojo, el cual indica advertencia. Esta alarma es presentada en la Figura 12. 


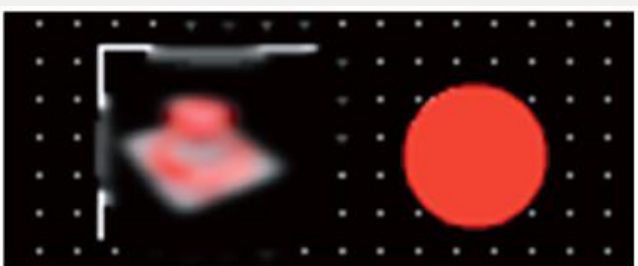

Figura 12. Paro de emergencia del proceso cervecero simulado

Fuente: Autores

\section{- Alarma de líquido en el tanque}

Esta alarma se activa y afecta únicamente a la etapa de llenado. Se activa cuando el tanque de cerveza está desocupado. Esta alarma es presentada en la Figura 13.

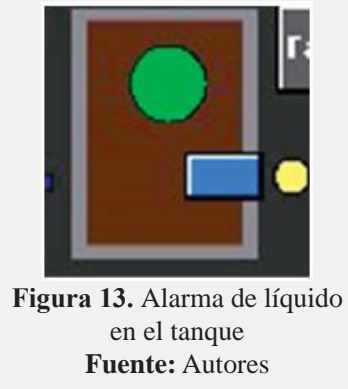

\section{- Alarma de tapas en el depósito}

Esta alarma se activa y afecta únicamente a la etapa de tapado. Se activa cuando no hay tapas en el depósito. Se muestra la alarma en la Figura 14.

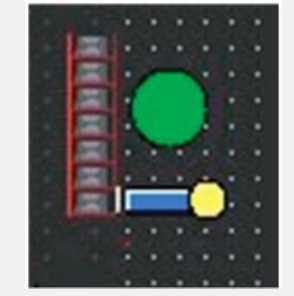

Jul - Dic 2016

ISSN 0122-820X

E-ISSN 2422-5053

PP: $57-70$

\subsubsection{Alarmas análogas}

Se implementaron tres alarmas análogas, mediante la función de avisos HMI. La primera alarma se activa cuando la temperatura del agua de pasteurizado está bajo el límite de $40^{\circ} \mathrm{C}$, la segunda alarma se activa cuando la temperatura del agua de pasteurizado está sobre el límite de $100^{\circ} \mathrm{C}$. La última alarma se activa cuando la velocidad del motor excede el límite de $90 \mathrm{~cm} / \mathrm{s}$. A continuación, en la Tabla IV se observa cómo se configuran los avisos HMI, para esto, se define el mensaje que se despliega en la pantalla cuando la alarma se activa, y se establece el valor límite de las variables. Si estas sobrepasan ese valor, ya sea por exceso o defecto, se alarma el proceso.

Tabla IV. Configuración de los avisos HMI para alarmas análogas en el software Tia Potal

\begin{tabular}{|c|c|c|c|c|c|}
\hline \multicolumn{6}{|c|}{ Avisos analógicos } \\
\hline ID & TEXTO DE AVISO & CATEGORÍA & VARIABLE DE DISPARO & VALOR LÍMITE & MODO DEL LÍMITE \\
\hline 1 & Temperatura bajo límite & Errors & tempemuestra & 40 & Rebase por defecto \\
\hline 2 & Temperatura sobre el límite & Errors & tempemuestra & 100 & Rebase por exceso \\
\hline 3 & Exceso de velocidad banda & Errors & gráfica & 90 & Rebase por exceso \\
\hline
\end{tabular}

Fuente: Autores

\subsection{Receta}

Para la simulación de las situaciones de emergencia, se realizaron cuatro recetas, para las cuales cambian los valores de temperatura a la cual se expone el agua para la pasteurización.
Lo primero que se hizo, fue darle nombre a la receta, y luego se agregó el ingrediente o elemento de esta, es decir, la temperatura, tal como se observa en la Tabla V. 
No. 2

Jul - Dic 2016 ISSN 0122-820X E-ISSN 2422-5053 PP: $57-70$

Tabla V. Elementos de las recetas en el software Tia Portal

\begin{tabular}{|c|c|c|c|c|c|c|c|}
\hline Receta & & & & & & & \\
\hline Nombre & $\begin{array}{c}\text { Nombre de } \\
\text { Visualización }\end{array}$ & Número & Versión & Tipo & $\begin{array}{l}\text { Número } \\
\text { máximo }\end{array}$ & $\begin{array}{c}\text { Tipo de } \\
\text { comunicación }\end{array}$ & Tooltip \\
\hline Receta 1 & Receta_1 & 1 & $27 / 05 / 2015$ & Limitado & 20 & Variables & \\
\hline & & & & & & & \\
\hline Elementos & Registros & & & & & & \\
\hline Nombre & $\begin{array}{c}\text { Nombre de } \\
\text { Visualización }\end{array}$ & Variable & Tipo de datos & Longitud & $\begin{array}{c}\text { Valor } \\
\text { predeterminado }\end{array}$ & Valor mínimo & $\begin{array}{c}\text { Valor } \\
\text { máximo }\end{array}$ \\
\hline $\begin{array}{c}\text { Temperatura } \\
\left({ }^{\circ} \mathrm{C}\right)\end{array}$ & temperatura & tempreffff & Int & 2 & 0 & -32768 & 32767 \\
\hline
\end{tabular}

Fuente: Autores

A continuación, se definieron las diferentes proporciones del ingrediente de la receta creada, para lo cual se determinaron cuatro clases o registros, las dos primeras corresponden al tipo de cerveza, y las otras dos a las temperaturas máxima y mínima del agua de pasteurizado, como se presenta en la Tabla VI.

Tabla VI. Registros de las recetas en el software TIA Portal

\begin{tabular}{|c|c|c|c|c|c|c|c|}
\hline \multirow{2}{*}{$\begin{array}{c}\text { Receta } \\
\text { Nombre }\end{array}$} & \multirow[b]{2}{*}{$\begin{array}{c}\text { Nombre de } \\
\text { Visualización }\end{array}$} & \multirow[b]{2}{*}{ Número } & \multirow[b]{2}{*}{ Versión } & \multirow[b]{2}{*}{ Tipo } & \multirow[b]{2}{*}{$\begin{array}{l}\text { Número } \\
\text { máximo }\end{array}$} & \multirow[b]{2}{*}{$\begin{array}{c}\text { Tipo de } \\
\text { comunicación } \\
\end{array}$} & \multirow[b]{2}{*}{ Tooltip } \\
\hline & & & & & & & \\
\hline Receta 1 & Receta_1 & 1 & $27 / 05 / 2015$ & Limitado & 20 & Variables & \\
\hline & & & & & & & \\
\hline Elementos & Registros & & & & & & \\
\hline Nombre & $\begin{array}{c}\text { Nombre de } \\
\text { Visualización }\end{array}$ & Número & $\begin{array}{c}\text { Temperatura } \\
\left({ }^{\circ} \mathrm{C}\right)\end{array}$ & Comentario & & & \\
\hline $\begin{array}{l}\text { Cerveza } \\
\text { Dorada }\end{array}$ & $\begin{array}{l}\text { Cerveza } \\
\text { Dorada }\end{array}$ & 1 & 350 & & & & \\
\hline Cerveza Roja & Cerveza Roja & 2 & 420 & & & & \\
\hline AlarmarBajo & Alarma Baja & 3 & 100 & & & & \\
\hline AlarmarAlto & Alarma Alta & 4 & 1500 & & & & \\
\hline
\end{tabular}

Fuente: Autores

\subsection{Visualización: Tres dimensiones}

Para la visualización 3D de todos los subprocesos, se utilizó el software Automation Studio 6. En la Figura 15 se puede observar la interfaz desarrollada, en la cual se ubicaron los distintos elementos que influyen en las etapas de llenado, tapado y pasteurizado, que van desde la banda transportadora, la botella, la electroválvula de llenado, el almacén de tapas, hasta el tanque de pasteurizado. En esta interfaz, se simula toda la dinámica del sistema por medio de animaciones, las cuales se ejecutan al mismo tiempo que los subprocesos implementados en el PLC. Lo anterior, gracias al servidor OPC de IBH, a través del cual se conecta el PLC con el software Automation Studio.

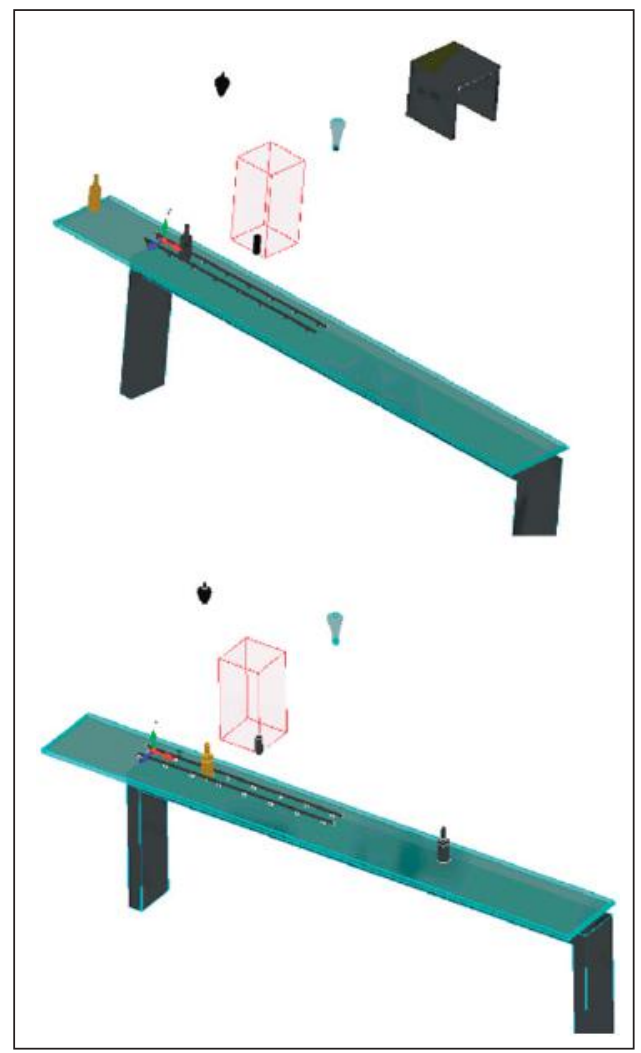

Figura 15. Intefaz desarrollada en Automation Studio que describe la dinámica del sistema Fuente: Autores 


\subsection{Monitoreo}

Para la supervisión y el control de todo el proceso de manera remota, se agregó una estación PC la cual contiene al módulo SIMATIC WinCC, que permite la supervisión.
Las conexiones del PLC con la estación PC, con la pantalla HMI, y con el motor de la banda transportadora, se pueden observar a continuación en la Figura 16.

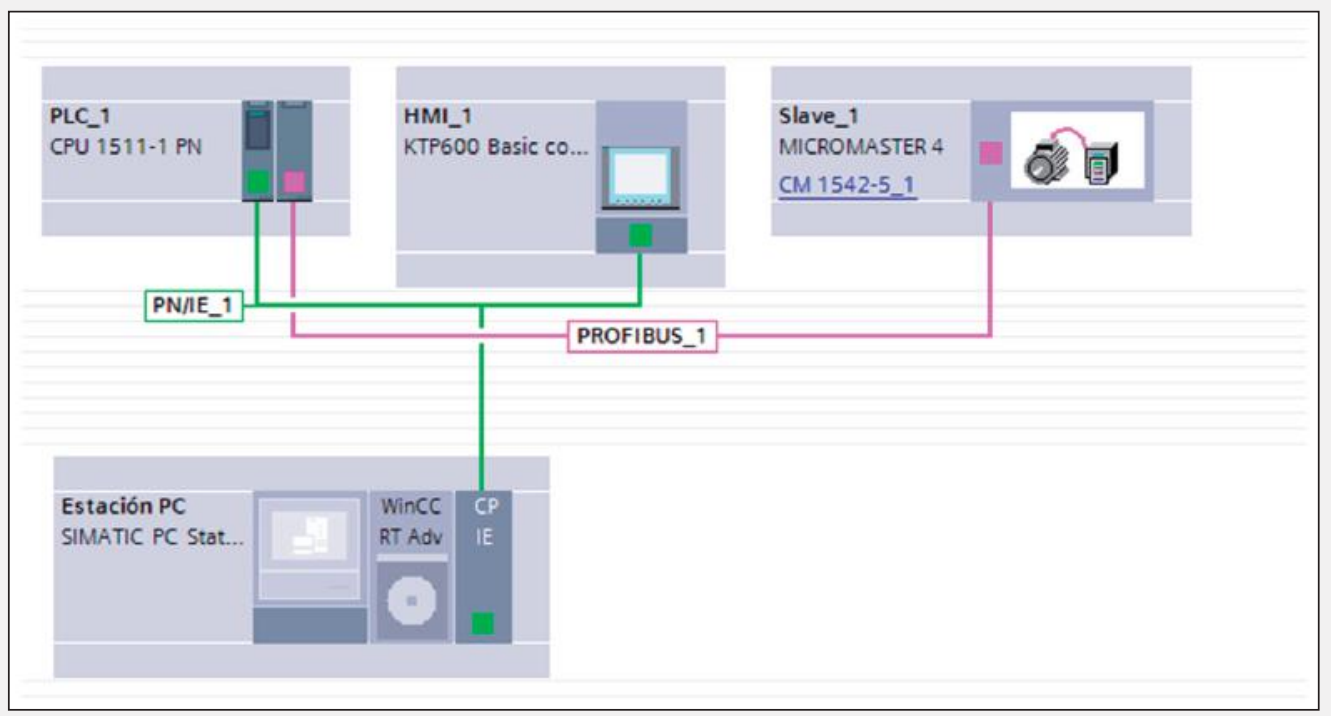

Figura 16. Diagrama de conexiones del sistema automatizado en el software TIA Portal Fuente: Autores

\section{Resultados y análisis}

La simulación realizada permite seguir paso a paso los subprocesos de interés, tanto de manera local como remota, permitiendo observar el comportamiento del sistema en condiciones normales $\mathrm{o}$ ante situaciones de emergencia a las cuales el simulador responde de manera inmediata. Lo anterior permite determinar si el proceso simulado es adecuado, y óptimo para automatizar una planta cervecera.

Respecto al sistema de fallas implementado, se observó que el uso de recetas fue útil para simular las situaciones de emergencia que se podrían presentar en la planta real, ya que con estas se le pudieron asignar distintos valores a las variables de disparo de las alarmas, por ejemplo, a la temperatura del agua de pasteurizado se le dieron dos valores, uno muy bajo, y uno muy alto, de manera tal que el usuario al selecionararlos le asignará dicho valor a la variable de disparo, este valor se encontraría por debajo o por encima de los límites permitidos, haciendo que la alarma se activara.

\section{Conclusiones}

La simulación realizada permite comprender el comportamiento de la planta cervecera, en cuanto a los procesos de llenado, tapado y pasteurizado, observando de manera detallada cada una de las etapas que conforman a los subprocesos.

El control on/off para la activación de los distintos sensores del proceso, es útil ya que este sistema se puede adaptar fácilmente a sensores reales. estándar de comunicación OPC es bueno debido a su rapidez, a que es actual, y a que acepta múltiples puertos.

Al momento de simular la planta en 3D se suelen presentar inconvenientes respecto a las coordenadas, ya que las del software TIA 
No. 2

Jul - Dic 2016 ISSN 0122-820X

E-ISSN 2422-5053

PP: $57-70$
Portal no concuerdan con las de Automation Studio.

Para el sistema de fallas, cuando una de las alarmas analógicas se activa, aparece un recuadro donde se le indica al usuario de esta. Dicho recuadro no desaparece de la pantalla HMI hasta que el usuario cambie los valores de las variables de disparo responsables, y desactive la alarma.

\section{Agradecimientos}

Los autores agradecen a la Universidad Militar Nueva Granada por el apoyo recibido durante la realización del proyecto.

\section{Referencias}

[1] Y. Bedoya, C. Salazar and J. Muñoz. Implementación, control y monitoreo de un sistema de seguridad vehicular por redes GSM/GPRS. Tesis de grado, Dept. Ingeniería Mecatrónica, Universidad Tecnológica de Pereira, Pereira, Colombia, 2013

[2] S. Iñiguez. "Automatización de procesos", 4 Octubre 2011. [Online]. Disponible en: https://es.over-blog.com/ Que_es_la_automatizacion_de_procesos-1228321767-art127041.html.

[3] Schneider Electric. PlantStruxure Sistema de automatización de procesos. [Online]. Disponible en: http://www. schneider-electric.com.mx/documents/ solutions/solution/PLANTSTRUXURE_
SCHC208.pdf.

[4] Universidad Nacional Experimental Politécnica. "Instrumentación y control", 14 Junio 2008. [Online]. Disponible en: http://instrumentacionycontrol.blogspot. com.co/2008/04/descargar-los-archivosde-clase-httpcid.html.

[5] M. Ogawa and Y. Henmi. "Recent Developments on PC+PLC based Control
Systems for Beer Brewery Process Automation Applications" SICE-ICASE International Joint Conference, pp. 10531056, 2006.

[6] Instituto tecnológico de Aragón. "Simulación de procesos", 2016. [Online]. Disponible en: http://web.itainnova.es/elogistica/lineas-de-trabajo/logistica-inteligente/ simulacion-de-procesos/.

[7] WEG. "Soluciones para automatización de procesos". [Online]. Disponible en: http://www.weg.net/co/Productos-y-Servicios/Drives/Automatizacion-de-Procesos-Industriales/Sistemas-de-Automatizacion-y-Control-de-Procesos.

[8] Z. Xiaodong, Z. Jie and L. Ke. "Design and implementation of control system for beer fermentation process based on SIMATIC PLC". The 27th Chinese Control and Decision Conference, 2015.

[9] Portafolio. "La industria cervecera de Colombia es de alta intensidad competitiva", 30 Mayo 2016. [Online]. Disponible en: http://www.portafolio.co/negocios/ empresas/industria-cervecera-colombiaalta-intensidad-competitiva-496595. 